\title{
Tweety Homologs (TTYH) Freshly Join the Journey of Molecular Identification of the VRAC/VSOR Channel Pore
}

\author{
Yasunobu Okada* \\ Department of Molecular Cellular Physiologye, Kyoto Prefectural University of Medicine, Kyoto 602-8566, \\ Division of Cell Signaling, National Institute for Physiological Sciences, Okazaki 444-8787, Japan
}

Cell volume regulation (CVR) is fundamental to the survival and functions of animal cells under physiological and pathological conditions. Two types of chloride channels are directly activated by cell swelling and involved in CVR. One is the volume-sensitive outwardly rectifying anion channel (VSOR) [1], also called the volume-regulated anion channel (VRAC) [2], which is ubiquitously expressed and exhibits the phenotypic properties $[1,2]$; and another is the maxi-anion channel (Maxi-Cl), the core component of which has recently been identified as SLCO2A1 [3]. As to the molecular identity of VSOR/VRAC (simply called VSOR hereafter), the first breakthrough was brought about by the discovery of involvements of LRRC8 members [4, 5]. In this issue of Experimental Neurobiology, Han et al. [6] makes the second breakthrough by demonstrating the involvement of Tweety homologs (TTYH) in the VSOR channel activity.

Two groups $[7,8]$ independently discovered the existence of functional VSOR in 1988. Since then, for a quarter of a century, its molecular identity had not been uncovered, despite much efforts of proposing and disproving many false-positive candidates, as summarized recently [9]. In 2014, two groups [4, 5] independently identified LRRC8A or SWELL1 as one of essential components of VSOR channel and reported on the same day. In addition, LRRC8C, $8 \mathrm{D}$ or $8 \mathrm{E}$ was later found to be required together with LRRC8A for functional VSOR activity $[5,10,11]$. The pore-forming roles of these LRRC8 members were suggested by discernible, though not drastic, alterations of ion selectivity by some point

\footnotetext{
* To whom correspondence should be addressed. TEL: 81-564-59-5262, FAX: 81-564-59-5263 e-mail:okada@nips.ac.jp
}

mutations and evidenced by successful reconstitution of anionic channel activity as well as by recent cryo-EM structure studies with purified LRRC8s, as summarized recently [12,13]. However, it must be pointed out that several important properties (such as their cytoplasmic ATP independence, smaller single-channel conductance, and little voltage-dependent inactivation kinetics) of LRRC8-reconstituted channels are distinct from those of native VSOR channel, as summarized recently $[9,14]$. More importantly, the channel reconstituted with purified LRRC8A plus 8D or 8E was activated by reduction of ionic strength $(\Gamma)$, but not by inflation-induced membrane expansion [15]. In addition, a reduced cytoplasmic $\Gamma$ was found to be required to activate the channels in HEK293 cells expressing LRRC8A alone or LRRC8A plus 8C [16]. In contrast, native VSOR channels can be activated by cell swelling-associated expansion of membrane infoldings [1] and inflation or membrane expansion by forcing fluid into the cells without any $\Gamma$ reduction [17] (also see [12]). Thus, it is highly likely that the LRRC8 channel is not the membrane expansion-activated volume-regulatory anion channel (Me-VRAC or VRAC swell $[6]$ ), but the ionic strength-sensitive volume-regulatory anion channel (Is-VRAC or VRAC ${ }_{\Gamma}[6]$ ). On the other hand, it is also evident that LRRC8 members play some roles in swelling-induced activation of anion channel currents exhibiting VSOR phenotypes, because swelling-activated VSOR currents were never observed in cells lacking all five LRRC8 genes but rescued by expressing LRRC8A together with LRRC8C or 8E [5], and because coinjection of LRRC8A RNA together with LRRC8D or 8E RNA gave rise to hypotonicity-induced VSOR currents in Xenopus oocytes which lack all LRRC8 genes and endogenous VSOR activity [18]. However, it 
must be noted that overexpression of LRRC8A plus 8C failed to increase swelling-induced VSOR currents above the endogenous level of VSOR currents in wild-type HEK293 and HCT116 cells [5]. Furthermore, cisplatin-resistant KCP-4 cells which exhibited distinctly smaller VSOR activity showed similar gene expression levels of all LRRC8 members to those in the parental KB cells as well as in other three different human epithelial cells $[9,19]$. Moreover, overexpression of LRRC8A plus $8 \mathrm{D}$ or $8 \mathrm{E}$ in KCP- 4 cells failed to restore VSOR activity up to the level in its parental $\mathrm{KB}$ cells [19]. Taken together, it appears that some as-yet-unidentified pore-related component other than LRRC8 members is required for VSOR channel activity. Thus, through the upsurge of LRRC8 studies for the past five years, the field has been faced with the questions: What is the missing component for the VSOR pore and how LRRC8 members are precisely involved in the VSOR activation mechanism? Now, C. Justin Lee's group [6] reports a pioneering research in astrocytes to answer these questions.

By surprise, Han et al. [6] observed that Lrrc8a shRNA and/ or Lrrc8c shRNA never suppressed the peak amplitude, though slowed the activation time course, of hypotonicity-induced VSOR currents in mouse astrocytes and human HEK293 cells when exposed to Tris-Cl-rich extracellular and intracellular solutions that do not contain cation channel-permeable small cations, $\mathrm{Na}^{+}$ and $\mathrm{K}^{+}$, but with only a trace amount of $\mathrm{Cs}^{+}$. On the other hand, they also observed that Lrrc8a-shRNA suppressed VSOR currents in HEK293 cells under NaCl-rich conditions but not in HEK293 cells overexpressing TTYH1 and AQP4 genes. These observations are in contrast to the previous observations made by other groups in astrocytes, as follows. Treatment with Lrrc8a-shRNA and $\operatorname{Lrc} 8 \mathrm{a}$-siRNA nearly abolished hypotonicity-induced VSOR currents in rat astrocytes under NMDG-Cl-rich and CsCl-rich conditions, respectively [20,21], and VSOR currents activated by hypotonic stimulation or by glutamate-induced GPCR stimulation were largely, though not completely, abolished in astrocytes derived from astrocyte-specific Lrrc8a (Swell1) knockout mice under intracellular CsCl-rich and extracellular NMDG-Cl-rich conditions [22]. In place of LRRC8 members, Han et al. [6] paid attention to an involvement of Tweety homologs, TTYH1, TTYH2 and TTYH3, because TTYH1/2/3 genes are highly expressed in astrocytes [23], and because TTYH1 was previously proposed as a candidate molecule for another volume-activated Maxi-Cl channel [24], though later disproved [9]. Actually, Ttyh1/2/3-shRNA virtually abolished hypotonicity-induced VSOR currents in mouse astrocytes in culture and in hippocampal CA1 slices under Tris-Cl-rich conditions [6]. Furthermore, Han et al. [6] strongly suggested TTYH1 as a VSOR pore-forming component, based on the following two observations. First, overexpression of the charge-neutralizing R165A mutant of TTYH1 or the R164A mutant of TTYH2 largely suppressed VSOR currents with reducing $\mathrm{Cl}^{-}$permeability in HEK293 cells under Tris-Cl-rich conditions. Second, VSOR currents in HEK293 cells overexpressing R165CTTYH1 was substantially sensitive to MTSES under Tris-Cl-rich conditions. In light of these results, as concluded by Han et al. [6], it is likely that the TTYH family represents bona fide Me-VRAC channel in astrocytes.

Like the last breakthrough with LRRC8, this time starting with Tweety homologs has opened novel enigmas to be unraveled in future studies. Why is astrocytic VSOR activity not sensitive to gene silencing of LRRC8A under Tris-Cl-rich conditions, although it was sensitive to the gene silencing under NMDG-Cl-rich conditions free of small cations? How is CVR exactly attained by the indirect interaction between LRRC8 and TTYH members (such as the model proposed in Fig. 12 [6]) in astrocytes? What about an involvement of the direct protein-protein interaction between LRRC8 and TTYH members in the CVR mechanism in astrocytes? What/how about an involvement of any TTYH member in swelling-independent VSOR activation (see [14, 25]) induced by GPCR stimulation and by reactive oxygen species (ROS)? What about an involvement of any TTYH member in VSOR activity in non-astrocytic cells? What is an alternative molecule involved in Me-VRAC activity in non-astrocytic cells, such as human epithelial cells, which do not significantly express TTYH genes? Lastly, to precisely verify the pore-forming roles of $\mathrm{LRRC} 8 \mathrm{~A} / \mathrm{C} / \mathrm{D} / \mathrm{E}$ in Is-VRAC and of TTYH1/2/3 in Me-VRAC, drastic alterations in the anion selectivity Eisenman's sequence and in the anion/cation permeability ratio must be observed with some charge-modifying (especially charge-reversing) mutations. At last, the VRAC/VSOR journey still continues with Tweety's surprising debut in the realm of molecular identification of the pore, following the recent LRRC8 upsurge.

\section{REFERENCES}

1. Okada Y (1997) Volume expansion-sensing outward-rectifier $\mathrm{Cl}^{-}$channel: fresh start to the molecular identity and volume sensor. Am J Physiol 273:C755-C789.

2. Nilius B, Eggermont J, Voets T, Buyse G, Manolopoulos V, Droogmans G (1997) Properties of volume-regulated anion channels in mammalian cells. Prog Biophys Mol Biol 68:69119.

3. Sabirov RZ, Merzlyak PG, Okada T, Islam MR, Uramoto H, Mori T, Makino Y, Matsuura H, Xie Y, Okada Y (2017) The organic anion transporter SLCO2A1 constitutes the core component of the Maxi-Cl channel. EMBO J 36:3309-3324. 
4. Qiu Z, Dubin AE, Mathur J, Tu B, Reddy K, Miraglia LJ, Reinhardt J, Orth AP, Patapoutian A (2014) SWELL1, a plasma membrane protein, is an essential component of volumeregulated anion channel. Cell 157:447-458.

5. Voss FK, Ullrich F, Münch J, Lazarow K, Lutter D, Mah N, Andrade-Navarro MA, von Kries JP, Stauber T, Jentsch TJ (2014) Identification of LRRC8 heteromers as an essential component of the volume-regulated anion channel VRAC. Science 344:634-638.

6. Han YE, Kwon J, Won J, An H, Jang MW, Woo J, Lee JS, Park MG, Yoon BE, Lee SE, Hwang EM, Jung JY, Park H, Oh SJ, Leec CJ (2019) Tweety-homolog (Ttyh) family encodes the pore-forming subunits of the swelling-dependent volumeregulated anion channel (VRACswell) in the brain. Exp Neurobiol 28:181-214.

7. Hazama A, Okada Y (1988) $\mathrm{Ca}^{2+}$ sensitivity of volume-regulatory $\mathrm{K}^{+}$and $\mathrm{Cl}^{-}$channels in cultured human epithelial cells. J Physiol 402:687-702.

8. Cahalan MD, Lewis RS (1988) Role of potassium and chloride channels in volume regulation by $\mathrm{T}$ lymphocytes. Soc Gen Physiol Ser 43:281-301.

9. Okada Y, Okada T, Islam M R, Sabirov RZ (2018) Molecular identities and ATP release activities of two types of volumeregulatory anion channels, VSOR and Maxi-Cl. Curr Top Membr 81:125-176.

10. Lutter D, Ullrich F, Lueck JC, Kempa S, Jentsch TJ (2017) Selective transport of neurotransmitters and modulators by distinct volume-regulated LRRC8 anion channels. J Cell Sci 130:1122-1133.

11. Sato-Numata K, Numata T, Inoue R, Sabirov RZ, Okada Y (2017) Distinct contributions of LRRC8A and its paralogs to the VSOR anion channel from those of the ASOR anion channel. Channels (Austin) 11:167-172.

12. Strange K, Yamada T, Denton JS (2019) A 30-year journey from volume-regulated anion currents to molecular structure of the LRRC8 channel. J Gen Physiol 151:100-117.

13. König B, Stauber T (2019) Biophysics and structure-function relationships of LRRC8-formed volume-regulated anion channels. Biophys J 116:1185-1193.

14. Okada Y, Okada T, Sato-Numata K, Islam MR, AndoAkatsuka Y, Numata T, Kubo M, Shimizu T, Kurbannazarova RS, Marunaka Y, Sabirov RZ (2019) Cell volume-activated and volume-correlated anion channels in mammalian cells: their biophysical, molecular, and pharmacological properties. Pharmacol Rev 71:49-88.

15. Syeda R, Qiu Z, Dubin AE, Murthy SE, Florendo MN, Mason
DE, Mathur J, Cahalan SM, Peters EC, Montal M, Patapoutian A (2016) LRRC8 proteins form volume-regulated anion channels that sense ionic strength. Cell 164:499-511.

16. Deneka D, Sawicka M, Lam AK, Paulino C, Dutzler R (2018) Structure of a volume-regulated anion channel of the LRRC8 family. Nature 558:254-259.

17. Hagiwara N, Masuda H, Shoda M, Irisawa H (1992) Stretchactivated anion currents of rabbit cardiac myocytes. J Physiol 456:285-302.

18. Gaitán-Peñas H, Gradogna A, Laparra-Cuervo L, Solsona C, Fernández-Dueñas V, Barrallo-Gimeno A, Ciruela F, Lakadamyali M, Pusch M, Estévez R (2016) Investigation of LRRC8-mediated volume-regulated anion currents in Xenopus oocytes. Biophys J 111:1429-1443.

19. Okada T, Islam MR, Tsiferova NA, Okada Y, Sabirov RZ (2017) Specific and essential but not sufficient roles of LRRC8A in the activity of volume-sensitive outwardly rectifying anion channel (VSOR). Channels (Austin) 11:109-120.

20. Elorza-Vidal X, Sirisi S, Gaitán-Peñas H, Pérez-Rius C, Alonso-Gardón M, Armand-Ugón M, Lanciotti A, Brignone MS, Prat E, Nunes V, Ambrosini E, Gasull X, Estévez R (2018) GlialCAM/MLC1 modulates LRRC8/VRAC currents in an indirect manner: Implications for megalencephalic leukoencephalopathy. Neurobiol Dis 119:88-99.

21. Formaggio F, Saracino E, Mola MG, Rao SB, Amiry-Moghaddam M, Muccini M,Zamboni R, Nicchia GP, Caprini M, Benfenati V (2019) LRRC8A is essential for swelling-activated chloride current and for regulatory volume decrease in astrocytes. FASEB J 33:101-113.

22. Yang J, Vitery MD, Chen J, Osei-Owusu J, Chu J, Qiu Z (2019) Glutamate-releasing SWELL1 channel in astrocytes modulates synaptic transmission and promotes brain damage in stroke. Neuron (in press).

23. Zhang Y, Chen K, Sloan SA, Bennett ML, Scholze AR, O'Keeffe S, Phatnani HP, Guarnieri P, Caneda C, Ruderisch N, Deng S, Liddelow SA, Zhang C, Daneman R, Maniatis T, Barres BA, Wu JQ (2014) An RNA-sequencing transcriptome and splicing database of glia, neurons, and vascular cells of the cerebral cortex. J Neurosci 34:11929-11947.

24. Suzuki M, Mizuno A (2004) A novel human $\mathrm{Cl}^{-}$channel family related to Drosophila flightless locus. J Biol Chem 279:22461-22468.

25. Akita T, Okada Y (2014) Characteristics and roles of the volume-sensitive outwardly rectifying (VSOR) anion channel in the central nervous system. Neuroscience 275:211-231. 